\title{
A Use and Exchange Perspective for Explicating the Notion of Service
}

\author{
Paul Johannesson ${ }^{(凶)}$ \\ Department of Computer and Systems Sciences, Stockholm University, \\ Stockholm, Sweden \\ pajo@dsv.su.se
}

The notion of service has proved elusive, in practice as well as in academic research. The lack of a common view of the notion of service makes it difficult to describe, classify and manage services in an efficient and effective way.

One attempt to defining the notion of service has focused on identifying properties (such as intangibility, inseparability, heterogeneity, and perishability) that distinguish services from other kinds of resources. While this approach offers advantages of simplicity and familiarity, it is problematic as the suggested properties are neither necessary nor sufficient. For example, not only services but also many other resources can be intangible, such as information and rights. The same observation holds for perishability and heterogeneity, with examples as fruits and handicraft objects.

Another approach for explicating the notion of service is to shift the attention to the use and exchange context of services. In this view, services are about enabling and regulating resource use and exchange within a community. For this purpose, services can provide convenient and flexible mechanisms that would not be attainable with a goods-oriented view.

The talk discusses three mechanisms for clarifying the notion of service based on a use and exchange perspective: rights distribution, abstraction and co-creation. First, services enable people to provide access to resources without transferring ownership but instead offering more restricted rights on them. Thereby, rights are distributed allowing for flexible arrangements of resource exchange. Secondly, services do not need to be specified in detail, in the sense that a service specification can focus on functionality and not structure. This abstraction allows for flexible arrangements with respect to the production and delivery of resources. Thirdly, services typically involve not only resources provided by the service supplier but also resources from the consumer, thereby allowing for co-creation. The talk also shows how these perspectives can be modelled using the REA ontology and how the approach can be compared to commitment-based views on services. 